\title{
Immediate and long-term transcriptional response of hind muscle tissue to transient variation of incubation temperature in broilers
}

Watcharapong Naraballobh', Nares Trakooljull', Eduard Muráni ${ }^{1}$, Ronald Brunner ${ }^{1}$, Carsten Krischek², Sabine Janisch ${ }^{3}$, Michael Wicke ${ }^{3^{\wedge}}$, Siriluck Ponsuksili ${ }^{1}$ and Klaus Wimmers ${ }^{{ }^{*}}$

\begin{abstract}
Background: In oviparous species accidental variation of incubation temperatures may occur under natural conditions and mechanisms may have evolved by natural selection that facilitate coping with these stressors. However, under controlled artificial incubation modification of egg incubation temperature has been shown to have a wide-ranging impact on post-hatch development in several poultry species. Because developmental changes initiated in-ovo can affect poultry production, understanding the molecular routes and epigenetic alterations induced by incubation temperature differences may allow targeted modification of phenotypes.

Results: In order to identify molecular pathways responsive to variable incubation temperature, broiler eggs were incubated at a lower or higher temperature $\left(36.8^{\circ} \mathrm{C}, 38.8^{\circ} \mathrm{C}\right)$ relative to control $\left(37.8^{\circ} \mathrm{C}\right)$ over two developmental intervals, embryonic days (E) 7-10 and 10-13. Global gene expression of M. gastrocnemius was assayed at E10, E13, and slaughter age [post-hatch day (D) 35] (6 groups; 3 time points; 8 animals each) by microarray analysis and treated samples were compared to controls within each time point. Transcript abundance differed for between 113 and 738 genes, depending on treatment group, compared to the respective control. In particular, higher incubation temperature during E7-10 immediately affected pathways involved in energy and lipid metabolism, cell signaling, and muscle development more so than did other conditions. But lower incubation temperature during E10-13 affected pathways related to cellular function and growth, and development of organ, tissue, and muscle as well as nutrient metabolism pathways at D35.

Conclusion: Shifts in incubation temperature provoke specific immediate and long-term transcriptional responses. Further, the transcriptional response to lower incubation temperature, which did not affect the phenotypes, mediates compensatory effects reflecting adaptability. In contrast, higher incubation temperature triggers gene expression and has long-term effects on the phenotype, reflecting considerable phenotypic plasticity.
\end{abstract}

Keywords: Gene expression, Pathway analysis, In-ovo development, Poultry, Microarray

\footnotetext{
* Correspondence: wimmers@fbn-dummerstorf.de

'Deceased

'Leibniz Institute for Farm Animal Biology (FBN), Institute for Genome

Biology, 18196 Dummerstorf, Germany

Full list of author information is available at the end of the article
} 


\section{Background}

Chickens and other birds are homeotherms that require that their body temperatures are maintained within a limited range during pre- and post-hatch processes [1]. Altering the temperature range during the critical developmental periods may cause only minor morphological differences, or could even produce lethal events. Since under natural conditions unpredictable periods may occur when incubation temperatures are unfavorable, natural selection could have promoted traits and mechanisms that provide resilience against such exogenous factors and that are reflected by immediate, acute or long-term, delayed responses (Du and Shine, 2015). Shifts in the incubation temperature of eggs under controlled experimental conditions have been shown to impact post-hatch development in several bird species. However, results of previous studies are inconsistent. For example, a higher incubation temperature was concluded to positively affect breast meat yield in featherless broilers [2] and muscle fiber size in turkey [3], but was associated with body weight loss in live chicken [4]. Similarly, lower incubation temperature was indicated to have a prolonged effect on female embryo mortality in Australian Brush-turkey [5], but reportedly reduced growth rates of wood duck [6]. Thus, the effects of incubation temperature changes on post-hatch development remain unclear. In particular, there is a lack of studies addressing the response to exogenous physical effects on the level of gene expression that will promote the understanding of the underlying compensatory, adaptive and regulatory process that might be associated with the treatment.

The in-ovo development of birds offers a valuable model in which to study environmental effects on myogenesis. Indeed, the identification of shifts in muscle and growth traits facilitates the detection of candidate genes for these traits. During avian myogenesis, the muscle fibers are formed in two phases. The primary muscle fiber, which is a core fiber, transforms to a myotube between the $4^{\text {th }}$ and $7^{\text {th }}$ embryonic days (E). Next, secondary muscle fibers, which are smaller and derive from myoblasts, arrange around the primary muscle fiber as a scaffold, proceeding until E15 [7]. Fetal myoblasts are most abundant between E8 and E12 [8]. After the secondary phase, depending on morphology and localization of the myofibers, the adult myoblasts will transform and become the primary source of myogenic precursors for postnatal muscle formation [9-11]. During both critical stages, temperature manipulation may cause differential expression of genes to produce phenotypic changes. Previous studies showed that elevated incubation temperature over E7-10 positively influenced carcass traits in broiler males, but did not affect meat quality [12]. Thus, shifting the incubation temperature during targeted periods of in-ovo development could contribute to the improvement of the efficiency of broiler meat production, without sacrificing meat quality. This study addresses the transcriptomic response of skeletal muscle tissue to transient reduction and elevation of incubation temperature at early (E7-10) and late (E10-13) secondary muscle fiber development. Microarray expression profiles of treated samples were compared to those of the respective controls immediate after the treatment periods (E7-10; E10-13) and also later at slaughter (Fig. 1). The results have implication for the molecular foundation of potential impact on meat production traits and also provide insight into the mechanisms involved in the resilience against low and the phenotypic plasticity against high incubation temperature.

\section{Results}

Global gene expression pattern of chicken hind muscle The chicken gene 1.0 ST array contains 165,815 probesets representing 20,828 transcripts encoding for 18,214 genes. After quality filtering and normalization, probesets representing 8,909 transcripts were subjected to

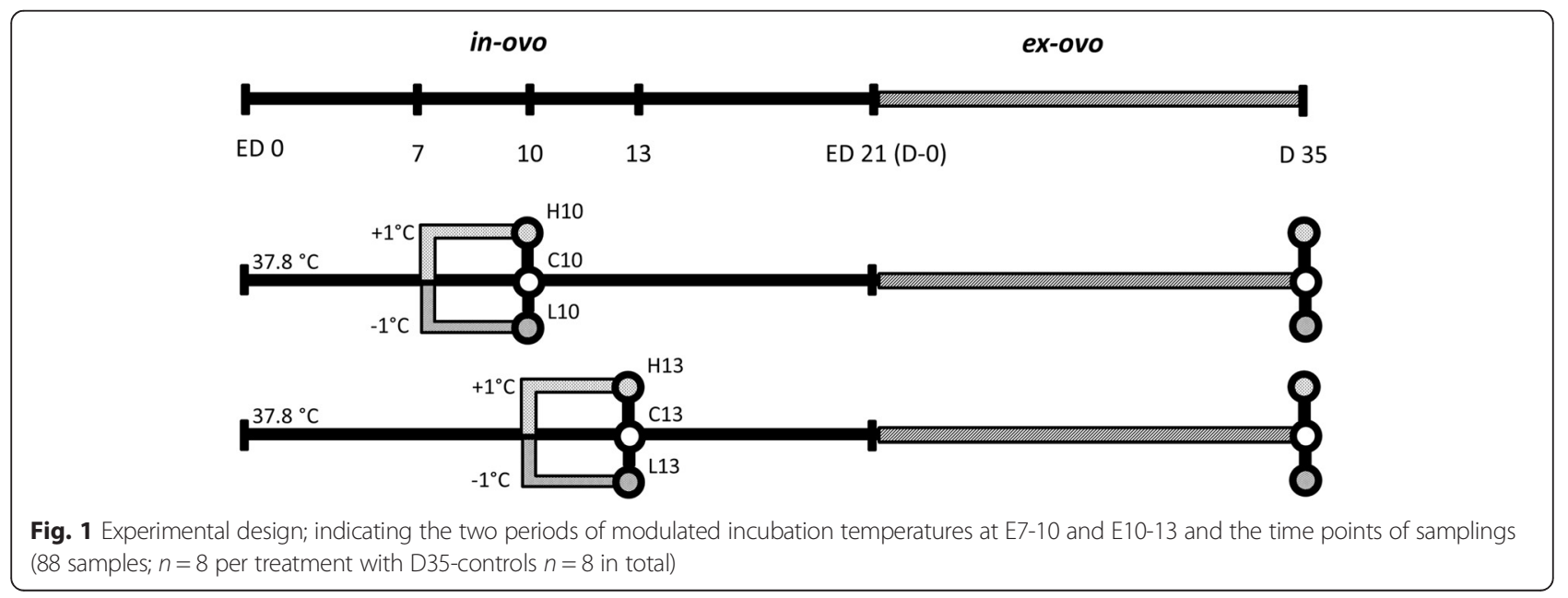


further analyses. Analysis of variance was used to identify differentially expressed genes (DEGs) by comparing gene expression levels of treatment group (in-ovo temperature modification) against the control. The number of DEGs for each comparison at embryonic stages and at D35 is shown in Table 1. At the embryonic stage, higher temperature during E7-10 versus control $(\mathrm{H} 10 \Delta \mathrm{C}$, $38.8^{\circ} \mathrm{C}$ ) significantly altered the expression of 738 genes compared to other treatment conditions (Table 1). Lower temperature during E10-13 versus control (L13 $\Delta$ C, $36.8^{\circ}$ C) affected more genes than did low temperature during E7-10 (389 vs 140). Long-term effects of the in-ovo temperature modification were investigated at D35. Lower temperature in the early and late treatment period (L10 $\Delta \mathrm{C}$ and L13 $\Delta$ C) resulted in a high number of DEGs at D35 (693 and 288, respectively), whereas higher temperature produced fewer DEGs at D35 (167 and 247, respectively) (Table 1). In addition, the majority of DEGs were downregulated in embryonic stage, but were up-regulated at D35, as shown in Table 1. The direction of regulation of FGA, NR4A3 and AHSG, exemplarily chosen as to represent genes assignment to several pathways, as indicated by microarrays and qPCR were consistent. The correlation coefficients were highly significant and ranged between 0.71 and 0.84 . Taken together, the qPCR analyses indicated a reproducible analysis.

\section{Distinct response to temperature alteration by time and direction}

A comparison of the DEGs between treatment conditions showed that most DEGs were unique for each condition, e.g., 685 and 366 DEGs for H10 $\Delta$ C and L13 $\Delta C$ in the embryonic stages (Fig. 2a), and 516 and 216 DEGs for L10 $\Delta$ C and L13 $\Delta$ C at D35 (Fig. 2b), respectively. Some DEGs were shared between two conditions, including those common either to L10 and L13 (10 and 45 at embryonic stage and D35) or to H10 and H13 (17 and 9 at embryonic

Table 1 Numbers of differentially expressed probes sets and respective genes (DEGs); comparisons between each in-ovo thermal modification condition the time-matched control separated for embryonic stages or at D35 ( $p \leq 0.05)$

\begin{tabular}{|c|c|c|c|c|c|}
\hline & \multirow{2}{*}{$\begin{array}{l}\text { Treatment } \\
(\Delta \mathrm{C})\end{array}$} & \multirow{2}{*}{$\begin{array}{l}\text { Probe } \\
\text { sets }\end{array}$} & \multirow[t]{2}{*}{ DEGs } & \multicolumn{2}{|c|}{ Regulation } \\
\hline & & & & Up & Down \\
\hline \multirow[t]{4}{*}{ Embryo } & $\mathrm{H} 10-\mathrm{C} 10$ & 812 & 738 & 662 & 76 \\
\hline & $\mathrm{H} 13-\mathrm{C} 13$ & 176 & 113 & 88 & 25 \\
\hline & L10 - C10 & 169 & 140 & 34 & 106 \\
\hline & L13 - C13 & 503 & 389 & 258 & 131 \\
\hline \multirow[t]{4}{*}{ D35 } & $\mathrm{H} 10-\mathrm{C} 10$ & 217 & 167 & 35 & 132 \\
\hline & $\mathrm{H} 13-\mathrm{C} 13$ & 332 & 247 & 108 & 139 \\
\hline & L10 - C10 & 768 & 693 & 104 & 589 \\
\hline & L13 - C13 & 330 & 288 & 123 & 165 \\
\hline
\end{tabular}

stage and D35), which were almost exclusively consistently regulated. There were only a few DEGs that were common across more than 2 conditions. Comparisons of DEGs from identical treatments in embryonic and D35 samples (Fig. 2c) revealed 55 common DEGs in total for the 4 treatments. Of these, 5 up- and 14 down-regulated transcript ids were regulated in the same direction in both embryonic stages and at D35. However, most of the common DEGs were regulated in opposite directions by stage, e.g., up-regulation in embryos and down-regulation at D35. A list of common DEGs with fold-change and $p$-value is available in Additional file 1.

\section{Pathway analysis}

To understand the underlying biology and identify relevant pathways, DEGs were analyzed using the Ingenuity Pathway Analysis software tools (IPA, Ingenuity Systems Inc., Redwood City, USA). All DEGs lists were separated into up- or down-regulated genes for each comparison (temperature modification vs control). All significant biological pathways associated with $p$-values and gene members are available in Additional files 2, 3, 4 and 5. The pathway analysis approach is effective for handling a list of DEGs, and generates a list of biological terms/ pathways. To encompass most pathways affected by all treatment factors (temperature modification, embryonic stage, and growth stage), we grouped 52 and 49 significant pathways derived from embryos and D35 broiler, respectively, into eight major categories of interest: group (gr.) 1, cell maintenance, proliferation, differentiation and replacement; gr.2, organismal, organ, and tissue development; gr.3, nutrient metabolism; gr.4, genetic information and nucleic acid processing; gr.5, molecular transport; gr.6, cell signaling and interaction; gr.7, small molecule biochemistry; and gr.8, response to stimuli. Overall, biological pathways involved in cell growth (gr.1) and tissue development (gr.2) were affected by modification of incubation temperature both in embryos and at D35 based on the number of pathways, as shown in Fig. 3. Activation states of upstream regulators were further analyzed for the dataset based on the Z-score calculation from Ingenuity Pathway Analysis (IPA) (Additional files 2-5).

In particular, a higher temperature during E7-10 (H10 $\Delta$ C; Fig. 3a, rows 1\&2) altered pathways involved in lipid metabolism, cell signaling, energy metabolism, muscle development and function, and small molecule biochemistry, more so than did other conditions in embryos (Fig. 3a, rows 3-8). Z-scores indicate that $\mathrm{H} 10 \Delta \mathrm{C}$ condition tended to activate several pathways related to nutrient metabolism (gr.3) and small molecule biochemistry (gr.7) (Additional file 2). A lower temperature (L10 $\Delta$ C and L13 $\Delta$ C; Fig. 3a, rows 5-8) affected pathways related cell maintenance, proliferation, differentiation and 

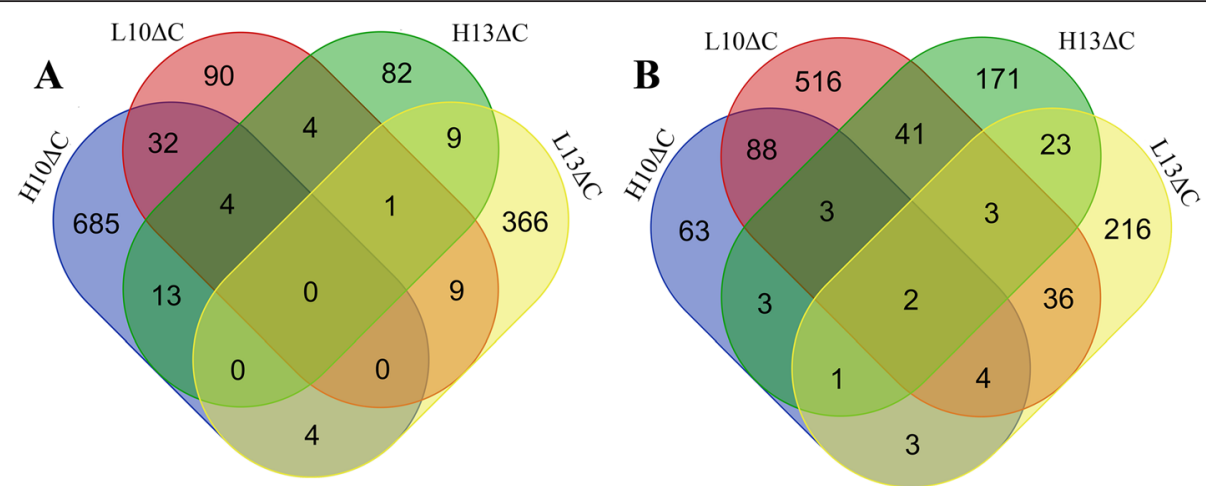

C
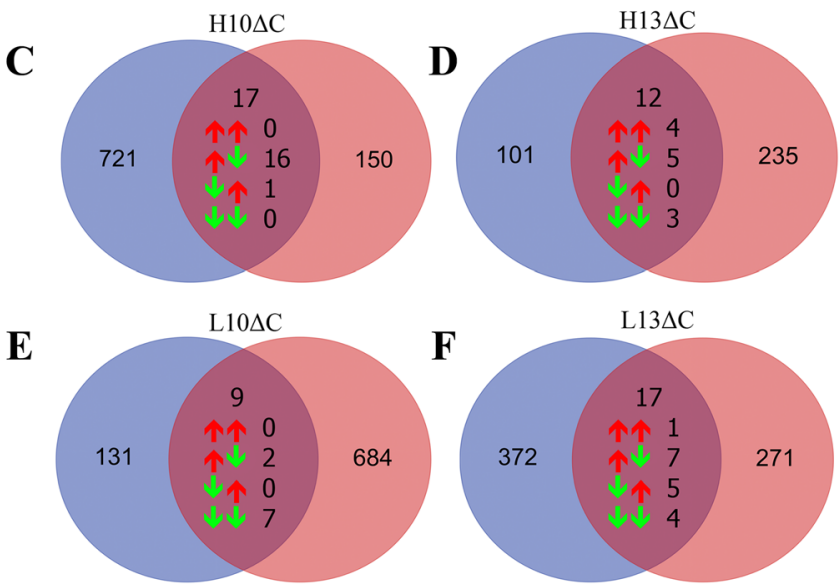

Fig. 2 Venn diagrams displaying numbers of differentially expressed genes for each treatment condition relative to control. Comparisons between treatment conditions at embryonic stages (a) and at D35 (b) as well as between embryonic stages and D35 after the same treatments (c) (blue embryonic, red D35)

replacement (gr.1) and organismal, organ, and tissue development (gr.2). L13 $\Delta \mathrm{C}$ tended to suppress cellar processes related to cell death, thus promoting maintenance in the major category cell maintenance, proliferation, differentiation and replacement (gr.1) and to activate developmental processes in mesoderm and muscle (gr.2, organismal, organ, and tissue development) (Additional file 3).

Using all DEGs obtained for $\mathrm{H} 10 \triangle \mathrm{C}$ in embryos, a network was generated covering 19 DEGs. FABP1 (fatty acid binding protein 1), PPARA (peroxisome proliferatoractivated receptor alpha), and PPARGC1A (peroxisome proliferator-activated receptor gamma, coactivator 1 alpha) are highly connected genes in the network and related to energy production, lipid metabolism and small molecule biochemistry (Fig. 4a). For L13 $\Delta$ C, the generated network was related to suppressed cell death and survival but stimulated cell growth and digestive developmental processes, including genes GPI (glucose-6-phosphate isomerase), NR1H3 (nuclear receptor subfamily 1 , group $\mathrm{H}$, member 3), and SRF (serum response factor) (Fig. 4b).

At D35, decreased incubation temperature during E10-13 (L13 $\Delta$ C; Fig. 3b, rows 7\&8) strongly changed pathways related to cellular function and growth development of organs, tissue and muscle as well as nutrient metabolism pathways (Fig. 3b). For L10 $\Delta$ C (Fig. 3b, rows 5\&6) a considerable number of genes were affected that belong to Ingenuity biological functions related to organismal, organ, and tissue development (gr.2). Notably, according to Z-scores L10 $\Delta \mathrm{C}$ tended to exhibit inhibitory effect on genetic processing categories (gr.4; Genetic information and nucleic acids), whereas $\mathrm{L} 13 \Delta \mathrm{C}$ was more likely to activate most categories (Additional file 4). For H13 $\Delta \mathrm{C}$ (Fig. 3b, rows $3 \& 4$ ) broadly the same molecular routes were shifted, however in opposite direction (Fig. 3b). For $\mathrm{H} 10 \Delta \mathrm{C}$, no trends of activation or inhibition of pathways were obvious (Additional file 5).

The network established for L10 $\Delta \mathrm{C}$ contained genes of top pathways including gene expression, cellular function and maintenance, and organismal development (Fig. 5a). Highly connected genes included HDAC4 (histone deacetylase 4), TBP (TATA Box Binding Protein), MYOD1 (myogenic differentiation 1), and SOX6 (sex determining region Y-box 6) that are related to inactivation of transcription and muscle cell differentiation. For $\mathrm{L} 13 \Delta \mathrm{C}$, activation of pathways related to proliferation, differentiation, and development at the cell, tissue, and organ levels was predicted. Accordingly, the consistently increased transcript abundances 


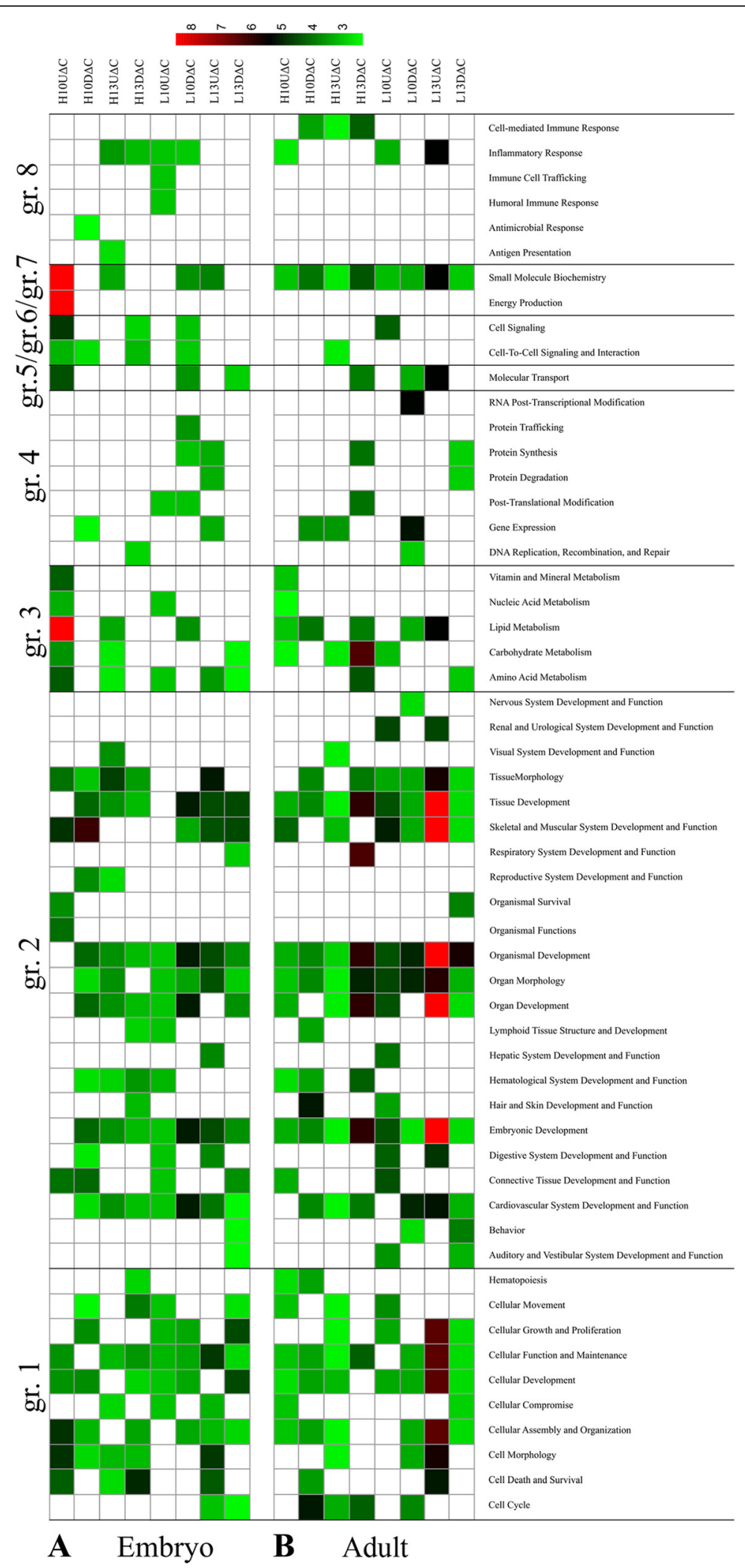

Fig. 3 (See legend on next page.) 
(See figure on previous page.)

Fig. 3 Significant pathways altered by in-ovo thermal modifications; in (a) embryonic stage and (b) D35. DEGs associated with each comparison (treatment vs control) are separated into up-regulation ( $U$ ) or down-regulation (D). Thermal modification treatments: increase $(H)$ or decrease $(L)$ incubation temperature during E7-10 (H10 and L10) or E10-13 (H13 and L13). Significant pathways (IPA defined) are grouped into eight major categories of interest; group (gr.)1 cell maintenance proliferation differentiation and replacement, gr.2 organismal organ and tissue development, gr.3 nutrient metabolism, gr.4 genetic information and nucleic acid processing, gr.5 molecular transport, gr.6 cell signaling and interaction, gr.7 small molecule biochemistry, and gr.8 response to stimuli and associated. The -log (BH p-value) associated with significant pathways are plotted in green (small) to red (large)

revealed a network (Fig. 5b). The involved genes for nutrition metabolism included APOA1 (apolipoprotein A1), GFPT1 (glutamine fructose-6-phosphate transaminase 1) and proliferation of muscle development included $A P O D$ (apolipoprotein D), and DES (desmin).

\section{Discussion}

This study demonstrates that transcriptomic and pathway regulation changes occur in broiler embryos and at D35 as a result of temperature manipulation during early (E7-10) and later (E10-13) development stage. Evidence was reported that early elevated incubation temperature positively influenced growth traits, but did not affect meat quality [12]. Indeed the chicken analyzed here showed slight but significant increase in body weight when transiently incubated at higher temperature, whereas decreased incubation temperature did not affect body weight.

\section{Immediate effects observed in-ovo}

Embryonic days 7-10 and 10-13 cover the final stage of primary muscle formation and start of secondary muscle fiber formation, respectively [7]. During myogenesis, multiple transcripts have major roles in regulating muscle development, such as $B C K$ ( $\mathrm{B}$ isoform of creatine kinase) [13], $c \operatorname{Tn} T$ (chicken cardiac troponin T) [14], Mstn (myostatin), and MyoD (myogenic differentiation 1) [15]. Previous research showed many regulated genes expressed during myogenesis being sensitive to incubation temperature manipulation.

\section{Immediate response to early high temperature treatment (H10 $\mathrm{C}$ )}

Due to our experiment, the major impacts on the transcriptome resulted from early high $(\mathrm{H} 10 \Delta \mathrm{C})$ and late low $(\mathrm{L} 13 \Delta \mathrm{C})$ temperature shifts, with the majority of DEGs being up regulated. The $\mathrm{H} 10 \Delta \mathrm{C}$ comparison indicated that

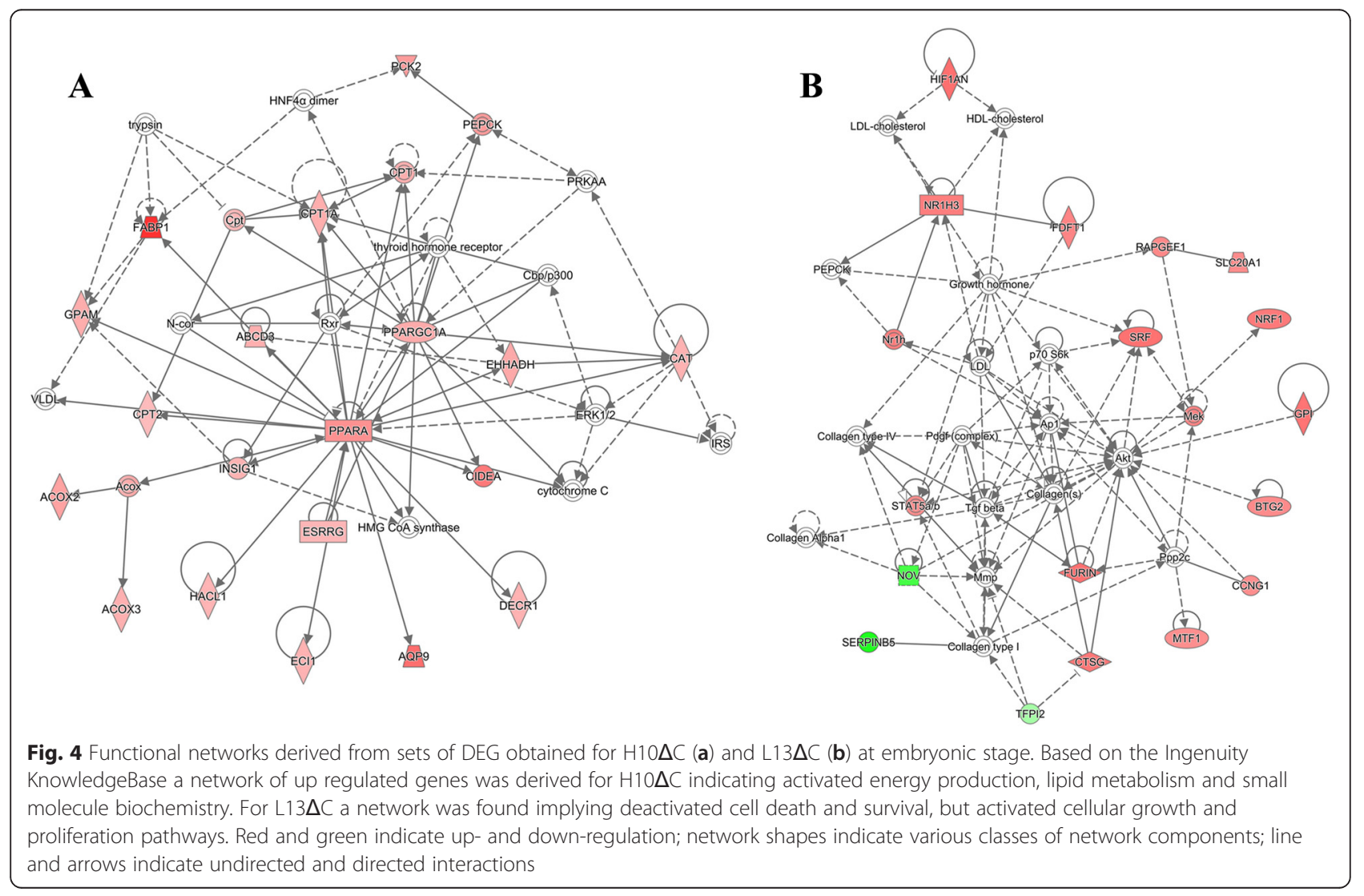



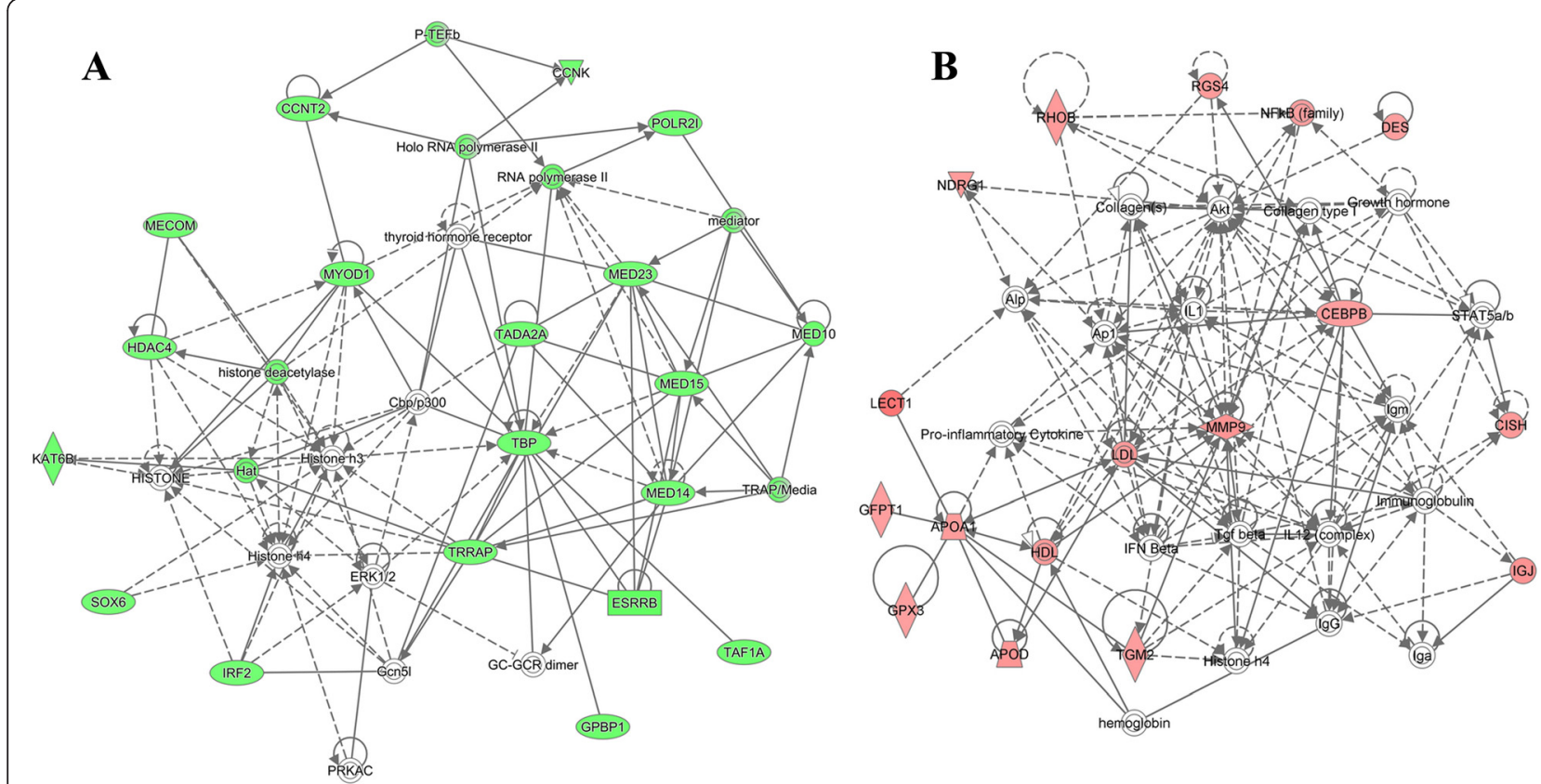

Fig. 5 Functional networks derived from sets of DEG obtained for $L 10 \Delta C$ (a) $L 13 \Delta C$ (b) at D35. Based on the Ingenuity KnowledgeBase a network of down regulated genes was derived for $\mathrm{L} 10 \Delta \mathrm{C}$ indicating deactivated gene expression, cellular function and organismal development networks. For $\mathrm{L} 13 \Delta \mathrm{C}$ a network was found implying deactivated tissue development, skeletal and muscular disorders and cell-to-cell signaling pathways. Red and green indicate up- and down-regulation; network shapes indicate various classes of network components; line and arrows indicate undirected and directed interactions

pathways involved in lipid metabolism, energy production, oxidation and beta-oxidation of fatty acid were activated. In this network, 19 up-regulated DEGs were represented, including FABP1, PPARA, and PPARGC1A.

$F A B P 1$ and PPARA act in fatty acid uptake, metabolism, and intracellular transportation of lipids metabolism, cell proliferation, cell differentiation and respiration as well as inflammation responses [16]. A previous study showed that $L-F A B P$ in chicken had higher expression in fat-type chicken at 3,5 , and 7 weeks old ( $p$-value $\leq 0.05$ ), and is linked to abdominal fat deposition and high lipogenesis rate [16]. Moreover, a previous study showed shifts of expression of $A M P K-P P A R A$ pathway genes due to thermal conductions [17]. PPARGC1, regulates energy metabolism, muscle fiber specialization and adaptive thermogenesis [18-20]. A previous study reported that single nucleotide polymorphisms (SNPs) in chicken PPARGC1A are significantly related to abdominal fat weight without growth trait effects [21]. Moreover, PPARGC1A (PGC-1 $\alpha)$ which was influenced by coldstress $\left(4{ }^{\circ} \mathrm{C}\right.$ from D28 to D38) in chicken could influenced a change in fiber type distribution and phenotype [20]. Exemplarily, modulated expressions of these genes reflect shifts of biological functions related to growth and metabolism.

The results demonstrate immediate shifts of transcript abundance due to manipulation of incubation temperature. During E7-10, high temperature $\left(38.8{ }^{\circ} \mathrm{C}\right)$ manipulation influenced mainly lipid (FABP1, PPARA) and energy production (PPARGC1) pathway. Accordingly, changes of body, liver, and heart weight were evident [22]. Moreover, activity of mitochondrial respiration (state-3-pyruvate/ma late and state-3-succinate/rotenone) and enzyme activities (glycogen phosphorylase, lactate dehydrogenase, and cytochrome oxidase) were elevated [22]. Thus, H10 treatment could influence lipid production and metabolism and also promoted phenotypic change.

\section{Immediate response to late low temperature treatment (L13 $\Delta \mathrm{C})$}

At later development (E10-13), low temperature had a greater impact on the transcriptome $(\mathrm{L} 13 \Delta \mathrm{C})$. The biological functions affected were predicted to affect cellular processes balancing differentiation, proliferation and maintenance. In contrast, high temperature treatment down regulated the development of cytoplasm and vasculogenesis, but increased inflammation and cell death. The L13 $\Delta$ C network related to cell death and survival and cellular growth and proliferation. Candidate DEGs included GPI, NR1H3, and SRF, involved in metabolic, proliferation, and differentiation pathways.

In fact GPI encodes a member of the glucose phosphate isomerase protein family, involved in glucose metabolism [23]. In chicken, GPI is up-regulated in muscle development [24]. NR1H3 belongs to the NR1 subfamily of the nuclear receptor superfamily (synonym: liver $\mathrm{X}$ 
receptor alpha), which are key regulators of macrophage function, inflammation, and lipid homeostasis in differentiating chondrocytes $[25,26]$. In chicken, $N R 1 H 3$ is considered a key regulator of fatty acid homeostasis [27] and cholesterol homeostasis [25]. SRF encodes a ubiquitous nuclear protein that stimulates cell proliferation and differentiation. In chicken embryo, SRF expression is restricted primarily to striated muscle cell lineages, which increased mass of nuclear and activating alphaactin gene activity [28].

Later in E10-13, lower temperature $\left(36.8^{\circ} \mathrm{C}\right)$ was associated with shifts of pathways towards balancing anabolic and catabolic pathways, which is in line with phenotypic change being slight and non-significant [22]. Enzyme activity (cytochrome oxidase) and mitochondrial respiration (state-3-pyruvate/malate) were lower than at normal condition. It was suggested that lower temperature at late treatment might decelerate embryonic activity.

\section{Long-term effects observed at D35}

In-ovo shifts of thermal conditions had long term effects on the transcriptome observed at D35. Higher or lower incubation temperature has also been shown to impact postnatal development in avian species [3, 6, 29]. Because the egg shell temperature is sensitive to environmental change, it can directly impact developmental processes as well [30,31]. Acute temperature modulation at the late embryonic stage was previously suggested to cause long-term transcriptomic changes, but few studies demonstrated an ongoing effect. A recent report showed embryonic temperature manipulation affected thermoregulatory mechanisms [32]. Another study found that periodic incubation temperature change between $37.8{ }^{\circ} \mathrm{C}$ and $39.5{ }^{\circ} \mathrm{C}$ from E16-18 initiated acute (E17) and lateterm (D13 post-hatch) positive effects on diameter of myofibers and muscle cell proliferation in chicken [1]. These findings might have been resulted from modifications to the stress response and thermogenesis by the increased temperature from E7-16, resulting in reduced oxygen consumption, heart rate, and egg shell temperature. These changes directly affected broiler embryo growth and development [33]. Similarly, another study showed that temperature manipulation caused a high density of blood vessels in the chorioallantoic membrane during embryogenesis [34]. In our studies, long-term transcriptomic changes were due to low temperature treatments (L10 $\Delta \mathrm{C}$ and L13 $\triangle \mathrm{C}$ conditions) primarily leading to downregulation. IPA analysis indicated that increasing the incubation temperature to $38.7^{\circ}$ may influence cell cycle and skin development at the early time point (E7-10). After that, high temperature E10-13 treatment tended to activate apoptosis in cell development but deactivated cardiovascular system and body trunk. Effects on metabolic process showed a reduction of carbohydrate metabolism, synthesis.
Furthermore, a negative effect still remained for concentration of lipid and acylglycerol.

\section{Long-term response to early low temperature treatment (L10 $\Delta$ C)}

The lower incubation temperature resulted in more DEGs in both early and late treatments. Early low temperature $(\mathrm{L} 10 \Delta \mathrm{C})$ tended to activate pathways involved in organismal development and cell proliferation but strongly suppress transcriptional process. All significant pathways including gene expression, cellular function and maintenance, and organismal development formed a network. Candidate genes included HDAC4, $M Y O D 1$, and SOX6, which are related to inactivation of transcription and muscle cell differentiation.

Previous research showed HDAC4 was associated in modulating cell growth and differentiation by controlling histone deacetylase activity, which alters chromosome structure and affects transcription factor access to DNA [35, 36]. A negative effect of $H D A C 4$ overexpression is down-regulation of cardiac muscle gene expression and leads to inhibition of cardiomyogenesis [37]. Normally HDAC4 was found in neuromuscular junction especially in myonuclei of fast oxidative skeletal muscle fibers [38, 39]. Down regulated of this gene suggested multiple transcriptional abnormalities including cardiac hypertrophy [40] and influence to MYOD1 expression. MYOD1 encodes a protein that belongs to a basic helix-loop-helix family of transcription factors and the myogenic factors subfamily. Generally, MYOD1 acts in muscle cell differentiation by inducing cell cycle arrest. During pre-gastrulating epiblast in chicken, $M Y O D 1$ can induce skeletal muscle lineage selfrenewal and differentiation [41]. Moreover, MYOD1 also works with the downstream effector $V G L-2$ in skeletal myogenesis [42]. Another DEGs which suggested to downregulate in cardiac and skeletal muscle is $S O X 6$, a member of the SOXD gene family, encodes functional domains including a DNA binding domain (the HMG box) and two coiled-coil domains [43]. The encoded protein is a transcriptional activator and critical role in cartilage development and mesenchymal differentiation [44]. Moreover, SOX6 is well known to function as a transcriptional suppressor of slow fiber-specific genes $[45,46]$.

Lowered incubation temperature had large effects on postnatal expression in terms of number of transcripts with shifted abundance. Manipulation of early treatment (E7-10) led to down regulation in transcriptional processes and muscle cell differentiation. Moreover, cardio (HDAC4) and skeletal (MYOD1 and SOX6) myogenesis were negatively affected. The phenotype of D35 chicken exposed to lower temperature showed a slight nonsignificant reduction of carcass and leg compared to the control group; higher incubation temperature led to increased weights (Additional file 6). 


\section{Long-term response to late low temperature treatment (L13 $\Delta \mathrm{C})$}

Late low temperature treatment $(\mathrm{L} 13 \Delta \mathrm{C})$ was predicted to activate pathways in cellular and organismal development including cell survival, development of body trunk, contractility of cardiac muscle, and proliferation of mammary epithelial cells, but to have a negative effect on size of body and muscle cell pathways. In metabolism, elevated uptake and metabolism of lipid and carbohydrate, together with small molecule biochemistry like oxidation of fatty acid, tended to reduce concentration of lipid. Inflammatory response was also predicted to be suppressed. The IPA network highlighted the activation of tissue development, skeletal and muscular disorders, and cell-to-cell signaling. Selection of Fold change (FC) related in every major category revealed a set of candidate genes: $A P O D, A P O A 1, D E S$, and GFPT1.

$A P O D$ encodes a component of high-density lipoprotein (HDL) with a high degree of homology to plasma retinolbinding protein and lipocalins. During late chicken embryogenesis, the expression of $A P O D$ is enriched among subsets of central nervous system (CNS) neurons then again in skin during developing of feather [47]. The molecular function involved lipoprotein metabolism, as shown by $A P O A 1$, $H D L$, and $L D L$ in the network Fig. $5 \mathrm{~b}$. $A P O A 1$ is the major protein component of high-density lipoprotein in plasma. It promotes cholesterol efflux from tissues to the liver for excretion, and is a cofactor for lecithin cholesterol acyl transferase (LCAT), which is responsible for the formation of most plasma cholesteryl esters. APOA1 is negatively correlated with aging and influences muscle development in Thai indigenous chicken [48]. Desmin (DES) encodes a muscle-specific class III intermediate filament. Homopolymers of this protein form a stable intra-cytoplasmic filamentous network connecting myofibrils to each other and to the plasma membrane. It maintains the structural integrity of highly solicited skeletal muscle and is important to other biological processes including muscle contraction and development, especially in heart contraction [49-51]. GFPT1 controls the flux of glucose into the hexosamine biosynthetic pathway, providing building blocks for the glycosylation of proteins and lipids [52]. The product of this gene catalyzes the formation of glucosamine 6-phosphate, which participates in carbohydrate biosynthesis and apoptosis regulation. GFPT1 is expressed in many tissues including skeletal muscle and heart [52]. Network connection revealed discreet interaction between GFPT1 and APOA1.

Low incubation temperature at late treatment (L13) had an impact on multiple DEGs and pathways in both embryo stages and at D35. However, these transcriptomic changes were not associated with significant phenotypic changes compared to the control (Additional file 6). The transcriptional response to lower incubation temperature appears to mediate compensatory effects that indicate a considerable adaptability. In nature transient reduction of incubation temperature during natural brooding happens. Accordingly, regulatory mechanism evolved in birds that enable the emergence of normal phenotypes. In contrast, higher incubation temperature triggers gene expression and has long-term effects on the phenotype. Elevated temperature is not likely in natural brooding, consequently not compensatory mechanisms evolved. Phenotypic changes associated with increased incubation temperature display metabolic plasticity of chicken.

\section{Conclusions}

Our experiment shows that manipulation of incubation temperature immediately effected transcriptomic changes and influenced the long-term expression. In parallel the results on growth, carcass, meat quality and mitochondrial respiratory activities indicate effects of transient variation of incubation temperature as well $[22,53]$. The observations indicate the successful activation of compensatory mechanisms in adaptation to lowered temperature and phenotypic plasticity in response to elevated temperature. Further investigations of the mechanism behind these regulatory processes including epigenetic modifications provide the perspective to improve resistance to environmental changes without much effect on growth performance [32]. Moreover, numerous genes which play important roles in metabolic pathways and which showed changed expression due to shifted incubation temperature represent candidate genes for further genetic improvement in terms of resilience against temperature shifts or in terms of increased muscle growth without affecting meat quality.

\section{Methods}

\section{Animals and tissue collection}

As outlined in Fig. 1, hatching eggs of a commercial broiler line (Cobb-Vantress Inc., Siloam Springs, USA) were randomly assigned to the following experimental groups: H10 and L10, which were subjected to higher $\left(38.8{ }^{\circ} \mathrm{C}\right)$ or lower $\left(36.8{ }^{\circ} \mathrm{C}\right)$ incubation temperature, respectively, between E7-10; and H13 and L13, which were subjected to the same temperature shifts, respectively, but between E10-13. During the rest of the incubation period, all eggs were incubated at $37.8{ }^{\circ} \mathrm{C}$, like the control group (C10, C13). Samples were collected immediately at the end of the treatment periods at E10 and E13, respectively, and in addition at post-hatch at day 35 . The hatchlings were reared in barn system and fed a standard diet ad libitum until day 35 (D35; slaughter). Samples of hind tissues (M. gastrocnemius) were collected and immediately stored in liquid nitrogen. Embryonic samples taken at ED10 and ED13 as well as samples of D35 were sexed and for each experimental group (C10, H10, L10 and C13, $\mathrm{H} 13, \mathrm{~L} 13)$ at each time point (E10 or E13, respectively, 
plus D35) samples, balanced for sex, were selected for gene expression analyses with 8 samples per treatment (Fig. 1). The recording of zoo-technical and biochemical traits was performed at the end of the respective treatment periods [53]. Increased incubation temperature led to slight but significant differences in body weight and mitochondrial respiratory capacity, whereas decreased incubation temperature only had subtle effects on a few parameters (Additional file 6). The study was approved by the institutional Animal Welfare Committees and was conducted according to the guidelines of the German Law of Animal Protection.

\section{RNA isolation}

Total RNA of frozen individual tissue samples was isolated with Tri-Reagent-extraction (Sigma-Aldrich, Taufkirchen, Germany) according to manufacturer's protocol. DNase treatment and a column-based purification using the RNeasy Mini Kit (Qiagen, Hilden, Germany) were also performed according to manufacturers' protocols. To check RNA integrity, samples were visualized on $1 \%$ agarose gels containing ethidium bromide. RNA concentration was determined by spectrometry with a NanoDrop ND-1000 spectrophotometer (PEQLAB, Erlangen, Germany). The absence of DNA contamination was confirmed by using the RNA as a template in standard PCR to amplify fragments of the glyceraldehyde-3-phosphate dehydrogenase (GAPDH) gene. To prevent degradation, all RNAs were stored at $-80{ }^{\circ} \mathrm{C}$ until further use.

\section{Expression microarray}

500 ng of total RNA was reverse-transcribed into cDNA with the Ambion WT Expression Kit (Life Technologies GmbH, Darmstadt, Germany). Biotin-labeled cRNA targets were made using the Affymetrix GeneChip WT Terminal Labeling Kit (Affymetrix, Santa Clara, CA, USA). Fragmented biotin-labeled cRNAs were hybridized onto Chicken Gene 1.0 ST Arrays (Affymetrix), which contains 18,214 probe-sets. After staining and washing, the arrays were scanned and raw data were obtained with the Affymetrix GCOS 1.1.1 software.

\section{Normalization and statistical analysis}

For expression data analysis raw data (cel-files) obtained by Affymetrix GCOS 1.1.1 software of all arrays were used as input files for the Affymetrix Expression Console for subsequent normalization and estimation of expression levels. Quantitative expression levels of transcripts were estimated using PLIER algorithm (Probe Logarithmic Intensity Error) and using DABG (detection above background) to evaluate detection by combining probe-level $p$-values to generate probe cell intensity values at exon level. All data were deposited in a MIAME-compliant database, the National Center for Biotechnology Information Gene Expression
Omnibus (www.ncbi.nlm.nih.gov/geo; accession number: GSE76670). All "present" values (default settings with detection $p$-values of $\leq 0.04$ ) were selected and integrated within gene-level annotation. To extract the outlying and nonspecific results, criteria on standard deviation $(\mathrm{SD} \geq$ $0.16)$ and means $(\mathrm{m} \geq 2.5)$ were applied using "genefilter" in $\mathrm{R}$ (www.r-project.org). Changes in transcript abundance were determined by analysis of variance (JMP Genomics, SAS-Institute) considering individual and combined effects of temperature, treatment period and gender and slaughter weight. Sex was excluded from the statistical model due to marginal effects. The final model included fixed effects of temperature, treatment period and interactions. Slaughter weight was included as covariate for the 35 days posthatched time course. Comparisons of treated samples ( $\mathrm{L}$ and $\mathrm{H}$ ) to controls $(\mathrm{C})$ within the respective time points (E10, E13, D35) were considered. Transcripts with significant differences of abundance at $p$-values $\leq 0.05$ were selected and queried for pathways analysis. At pre-hatch stages $p \leq 0.05$ equals FDR adjusted $p$-values of $\mathrm{q} \leq 0.18$; at D35 $p \leq 0.05$ corresponding q-values ranged between 0.35 and 0.70 .

\section{Real time quantitative RT-PCR (qPCR)}

For validation of microarray data, the gene expression of three genes was determined by Real-time quantitative PCRs using the same D35 samples used for microarray analyses. The assays were done in duplicate in volumes of $10 \mu \mathrm{l}$ using the LightCycler 480 SYBR Green I Master Kit (Roche), on a LightCycler 480 Real-Time PCR System (Roche Diagnostics GmbH, Germany). The temperature profiles comprised an initial denaturation step at $95^{\circ} \mathrm{C}$ for $10^{\prime}$ and 40 cycles consisting of denaturation at $95^{\circ} \mathrm{C}$ for 15 ", annealing at $60^{\circ} \mathrm{C}$ for $10^{\prime \prime}$ and extension at $72{ }^{\circ} \mathrm{C}$ for $15^{\prime \prime}$. The amplified genes were GAPH and ACTP as well as $F G A, N R 43 A$ and AHSG (Additional file 7), where the first two were used as reference genes to account for variation of cDNA amounts after reverse transcription by calculating a normalization factor. Target genes were selected because of their redundant assignment to different but related biofunctions. For all the assays threshold cycles were converted to copy numbers using a standard curve generated by amplifying serial dilutions of an external PCR standard $\left(10^{7}-10^{2}\right.$ copies). After completion of amplification protocol all samples were subjected to melting curve analyses and gel electrophoresis. Primers were obtained from Sigma-Aldrich, Germany.

\section{Pathway analyses and major categories}

Least-squares means of expression level and fold changes including "UP" and "DOWN" regulation among the tissues were estimated. Annotation data for Affymetrix Chicken Genome Arrays were obtained from the producer (Affymetrix Chicken Genome Array annotations release 
34). Ingenuity Pathway Analysis was used for functional annotation estimation of association between dataset and pathway. Differentially expressed genes (DEGs) were analyzed referring to Ingenuity Pathways Knowledge Base (IPKB). Biological and canonical pathways were identified from the IPKB library. Significance was considered based on Fisher's exact test $p$-values adjusted for multiple testing using the Benjamini-Hochberg correction procedure. Cutoff criteria were set to corrected $\mathrm{BH} p$-values $\leq 0.05$ for canonical pathways and for biofunctions, respectively. The variation of pathways was assigned, and we focused on the top most affected biological functions related to tissue development and myogenesis. All pathways were grouped into new categories based on criteria concerning the major roles in comprehensive biological routes on organismal, organ, tissue, cell or molecular levels. All biological functions were categorized in eight major groups (gr. 1 gr. 8) as follows: cell maintenance, proliferation, differentiation, and replacement (gr. 1); organismal organ and tissue development (gr. 2); nutrient metabolism (gr. 3); genetic information and nucleic acid processing (gr. 4); molecular transport (gr. 5); cell signaling and interaction (gr. 6); small molecule biochemistry (gr. 7); and response to stimuli (gr. 8). "Activated" and "deactivated" genes were assigned by positive and negative Z-scores, predicting the activation state of related transcription regulators. Significant pathways that were altered with in-ovo temperature modifications were clustered and visualized by heatmap. Genes assigned to major categories, as defined below, and with Z-scores were selected to derive IPA networks. Top network results were displayed covering related DEGs with annotation from NCBI reference sequence base [54].

\section{Availability of supporting data}

All supporting data are included in additional files.

\section{Additional files}

Additional file 1: Table S1. Common DEGs at embryonic stages and D35. Table S2. Common DEGs of H10 and H13 or L10 and L13 at embryonic stages. Table S3. Common DEGs of $\mathrm{H} 10$ and $\mathrm{H} 13$ or $\mathrm{L} 10$ and L13 at D35. (DOCX $41 \mathrm{~kb})$

Additional file 2: Assignment of DEGs to major categories, and biological functions obtained at embryonic stage for early treatment; H10U $\triangle C, H 10 D \Delta C, L 10 U \Delta C$ and L10D $\Delta C$. (DOCX $21 \mathrm{~kb}$ )

Additional file 3: Assignment of DEGs to major categories, and biological functions obtained at embryonic stage for late treatment; $\mathrm{H} 13 \cup \Delta \mathrm{C}, \mathrm{H} 13 \mathrm{D} \Delta \mathrm{C}$, L13U $\triangle \mathrm{C}$ and L13D $\Delta \mathrm{C}$. (DOCX $23 \mathrm{~kb})$

Additional file 4: Assignment of DEGs to major categories, and biological functions obtained at D35 for early treatment; $\mathrm{H} 10 \mathrm{U} \Delta \mathrm{C}$ $\mathrm{H} 10 \mathrm{D} \Delta \mathrm{C}$, L10U $\Delta \mathrm{C}$ and L10D $\Delta \mathrm{C}$ (DOCX $22 \mathrm{~kb}$ )

Additional file 5: Assignment of DEGs to major categories, and biological functions obtained at D35 for late treatment; $\mathrm{H} 13 \cup \Delta \mathrm{C}$ H13D $\Delta C$, L13U $\Delta C$ and L13D $\Delta C$. (DOCX $23 \mathrm{~kb})$
Additional file 6: Body weight, carcass weight and weight of hind muscles of broilers of the experimental groups used for expression analyses. (DOCX $19 \mathrm{~kb}$ )

Additional file 7: Primers used for quantitative real-time PCR ( $P P C R$ ) (DOCX $15 \mathrm{~kb})$

\section{Abbreviations}

$\Delta \mathrm{C}$ : 'delta' control, difference treatment versus control; D35: post-hatch days 35, slaughter date; DEGs: differentially expressed genes; E7, E10,

E13: embryonic days $7^{\text {th }}, 10^{\text {th }}$ and $13^{\text {th }}$ respectively; gr.1: major category group 1 cell maintenance, proliferation, differentiation and replacement; gr.2: major category group 2 organismal, organ, and tissue development; gr.3: major category group 3 nutrient metabolism; gr.4: major category group 4 genetic information and nucleic acid processing; gr.5: major category group 5 molecular transport; gr.6: major category group 6 cell signaling and interaction; gr.7: major category group 7 small molecule biochemistry; gr.8: major category group 8 response to stimuli; $\mathrm{H10}$ : embryos were incubated at high temperature $\left(38.8^{\circ} \mathrm{C}\right)$ at embryonic days $7-10$ and at control temperature $\left(37.8^{\circ} \mathrm{C}\right)$ at remaining time before and after;

$\mathrm{H} 13$ : embryos were incubated at high temperature $\left(38.8^{\circ} \mathrm{C}\right)$ at embryonic days 10-13 and at control temperature $\left(37.8^{\circ} \mathrm{C}\right)$ at remaining time before and after; IPA: Ingenuity Pathway Analysis; L10: embryos were incubated at low temperature $\left(36.8^{\circ} \mathrm{C}\right)$ at embryonic days $7-10$ and at control temperature $\left(37.8^{\circ} \mathrm{C}\right)$ at remaining time before and after; L13: embryos were incubated at low temperature $\left(36.8^{\circ} \mathrm{C}\right)$ at embryonic days $10-13$ and at control temperature $\left(37.8^{\circ} \mathrm{C}\right)$ at remaining time before and after.

\section{Competing interests}

The authors declare that they have no competing interests.

\section{Authors' contributions}

Conceived and designed the experiments: CK MW KW. Performed the experiments: WN CK SJ MW SP KW. Participated in sampling: WN RB CK SJ. Analyzed the data: WN NT EM SP KW. Drafted and wrote the manuscript: WN KW. Reviewed and approved the final manuscript: WN NT EM RB CK SJ SP KW.

\section{Acknowledgment}

This study was funded by the German Research Foundation (DFG; WE 4079/4-1, WI 1754/15-1, WI 2072/9-1).

\section{Author details}

${ }^{1}$ Leibniz Institute for Farm Animal Biology (FBN), Institute for Genome Biology, 18196 Dummerstorf, Germany. ${ }^{2}$ Institute of Food Quality and Food Safety, University of Veterinary Medicine Hannover, D-30173 Hannover, Germany. ${ }^{3}$ Department of Animal Science, Quality of Food of Animal Origin, Georg-August-University Goettingen, D-37075 Goettingen, Germany.

Received: 20 January 2016 Accepted: 25 April 2016

Published online: 04 May 2016

\section{References}

1. Piestun Y, Harel M, Barak M, Yahav S, Halevy O. Thermal manipulations in late-term chick embryos have immediate and longer term effects on myoblast proliferation and skeletal muscle hypertrophy. J Appl Physiol. 2008;106:233-40.

2. Hadad Y, Cahaner A, Halevy O. Featherless and feathered broilers under control versus hot conditions. 2. Breast muscle development and growth in pre- and posthatch periods. Poult Sci. 2014;93:1076-88.

3. Maltby $V$, Somaiya A, French NA, Stickland NC. In ovo temperature manipulation influences post-hatch muscle growth in the turkey. Br Poult Sci. 2004:45:491-8.

4. Krischek C, Gerken M, Wicke M. Effects of a higher incubation temperature between embryonic day 9 and 12 on growth and meat quality characteristics of turkeys. Br Poult Sci. 2013:54:5-11.

5. Eiby YA, Wilmer JW, Booth DT. Temperature-dependent sex-biased embryo mortality in a bird. Proc R Soc B Biol Sci. 2008:275:2703-6.

6. Durant SE, Hepp GR, Moore IT, Hopkins BC, Hopkins WA. Slight differences in incubation temperature affect early growth and stress endocrinology of wood duck (Aix sponsa) ducklings. J Exp Biol. 2010;213:45-51. 
7. Miller JB, Stockdale FE. What muscle cells know that nerves don't tell them. Trends Neurosci. 1987;10:325-9.

8. Stockdale FE. Myogenic cell lineages. Dev Biol. 1992;154:284-98.

9. Hartley RS, Bandman E, Yablonka-Reuveni Z. Skeletal muscle satellite cells appear during late chicken embryogenesis. Dev Biol. 1992;153:206-16.

10. Mauro A. Satellite cell of skeletal muscle fibers. J Biophys Biochem Cytol. 1961;9:493-5.

11. Schultz E, McCormick KM. Skeletal muscle satellite cells. Rev Physiol Biochem Pharmacol. 1994;123:213-57.

12. Werner $C$, Wicke $M$. Increasing the temperature at a certain period during the incubation of broiler eggs improves the carcass and breast yields without a negative impact on the meat quality. Arch Anim Breed. 2008;51:11-6.

13. Lyons GE, Mühlebach S, Moser A, Masood R, Paterson BM, Buckingham ME, Perriard JC. Developmental regulation of creatine kinase gene expression by myogenic factors in embryonic mouse and chick skeletal muscle. Dev Camb Engl. 1991;113:1017-29.

14. Tidyman WE, Sehnert AJ, Huq A, Agard J, Deegan F, Stainier DYR, Ordahl CP. In vivo regulation of the chicken cardiac troponin T gene promoter in zebrafish embryos. Dev Dyn. 2003;227:484-96.

15. Gabriel J, Alves H, Do Rosário M, Secatto A, Coutinho L, Macari M. Abundance of MyoD and myostatin transcripts in chicken embryos submitted to distinct incubation temperatures and timing exposures. Braz J Biol. 2011;71:563-4.

16. Zhang $Q$, Shi H, Liu W, Wang Y, Wang Q, Li H. Differential expression of LFABP and L-BABP between fat and lean chickens. Genet Mol Res. 2013;12: 4192-206.

17. Zhang Z, Bi M, Yao H, Fu J, Li S, Xu S. Effect of cold stress on expression of AMPKalpha-PPARalpha pathway and inflammation genes. Avian Dis. 2014; 58:415-26.

18. Lin J, Wu H, Tarr PT, Zhang C-Y, Wu Z, Boss O, Michael LF, Puigserver P, Isotani E, Olson EN, Lowell BB, Bassel-Duby R, Spiegelman BM: Transcriptional co-activator PGC-1 alpha drives the formation of slow-twitch muscle fibres. Nature. 2002;418:797-801

19. Shu JT, Xu WJ, Zhang M, Song WT, Shan YJ, Song C, Zhu WQ, Zhang XY, L HF. Transcriptional co-activator PGC-1a gene is associated with chicken skeletal muscle fiber types. Genet Mol Res. 2014;13:895-905.

20. Ueda M, Watanabe K, Sato K, Akiba Y, Toyomizu M. Possible role for avPGC-1a in the control of expression of fiber type, along with avUCP and avANT mRNAs in the skeletal muscles of cold-exposed chickens. FEBS Lett. 2005:579:11-7.

21. Wu GQ, Deng XM, Li JY, Li N, Yang N. A potential molecular marker for selection against abdominal fatness in chickens. Poult Sci. 2006;85:1896-9.

22. Krischek C, Janisch K, Naraballobh W, Brunner R, Wimmers K, Wicke M Alteration of the incubation temperature between embryonic day 7 and 13 influences weights and mitochondrial respiratory and enzyme activities in breast and leg muscles of broiler embryos. Mol Reprod Dev. 2016;83:71-8.

23. Rengaraj D, Lee SI, Yoo M, Kim TH, Song G, Han JY. Expression and knockdown analysis of glucose phosphate isomerase in chicken primordial germ cells. Biol Reprod. 2012;87:57.

24. Hallböök F, Persson H, Barbany G, Ebendal T. Development and regional expression of chicken neuroleukin (glucose-6-phosphate isomerase) messenger RNA. J Neurosci Res. 1989;23:142-51.

25. Gentili C, Tutolo G, Pianezzi A, Cancedda R, Descalzi Cancedda F. Cholesterol secretion and homeostasis in chondrocytes: a liver $\mathrm{X}$ receptor and retinoid $\mathrm{X}$ receptor heterodimer mediates apolipoprotein $\mathrm{A} 1$ expression. Matrix Biol. 2005;24:35-44.

26. Song C, Hiipakka RA, Kokontis JM, Liao S. Ubiquitous receptor: structures, immunocytochemical localization, and modulation of gene activation by receptors for retinoic acids and thyroid hormones. Ann N Y Acad Sci. 1995;761:38-49.

27. Demeure O, Duby C, Desert C, Assaf S, Hazard D, Guillou H, Lagarrigue S: Liver $\mathrm{X}$ receptor regulates fatty acid synthase expression in chicken. Poult Sci. 2009;88:2628-35

28. Croissant JD, Kim J-H, Eichele G, Goering L, Lough J, Prywes R, Schwartz RJ: Avian Serum Response Factor Expression Restricted Primarily to Muscle Cell Lineages Is Required for a-Actin Gene Transcription. Dev Biol. 1996:177:250-64.

29. Barri A, Honaker CF, Sottosanti JR, Hulet RM, McElroy AP. Effect of incubation temperature on nutrient transporters and small intestine morphology of broiler chickens. Poult Sci. 2011;90:118-25.
30. Hulet R, Gladys G, Hill D, Meijerhof R, El-Shiekh T. Influence of egg shell embryonic incubation temperature and broiler breeder flock age on posthatch growth performance and carcass characteristics. Poult Sci. 2007;86:408-12.

31. Joseph NS, Lourens A, Moran ET. The effects of suboptimal eggshell temperature during incubation on broiler chick quality, live performance, and further processing yield. Poult Sci. 2006;85:932-8.

32. Loyau T, Bedrani L, Berri C, Métayer-Coustard S, Praud C, Coustham V, Mignon-Grasteau S, Duclos MJ, Tesseraud S, Rideau N, HennequetAntier C, Everaert N, Yahav S, Collin A: Cyclic variations in incubation conditions induce adaptive responses to later heat exposure in chickens: a review. Animal. 2015;9:76-85.

33. Piestun $Y$, Halevy O, Yahav S. Thermal manipulations of broiler embryos-The effect on thermoregulation and development during embryogenesis. Poult Sci. 2009;88:2677-88.

34. Pinchuck S, Meiri N, Yahav S. The effect of thermal manipulation, during early embryogenesis, on angiogenesis and thermo-tolerance in broilers. Wageningen: IFRG; 2011. p. 44-5.

35. Backs J, Song K, Bezprozvannaya S, Chang S, Olson EN. CaM kinase II selectively signals to histone deacetylase 4 during cardiomyocyte hypertrophy. J Clin Invest. 2006;116:1853-64.

36. Guan Y, Chen Q, Yang X, Haines P, Pei M, Terek R, Wei X, Zhao T, Wei L: Subcellular relocation of histone deacetylase 4 regulates growth plate chondrocyte differentiation through Ca2+/calmodulin-dependent kinase IV. AJP Cell Physiol. 2012;303:C33-40.

37. Karamboulas C, Swedani A, Ward C, Al-Madhoun AS, Wilton S, Boisvenue S, Ridgeway AG, Skerjanc IS. HDAC activity regulates entry of mesoderm cells into the cardiac muscle lineage. J Cell Sci. 2006;119:4305-14.

38. Cohen TJ, Barrientos T, Hartman ZC, Garvey SM, Cox GA, Yao T-P. The deacetylase HDAC4 controls myocyte enhancing factor-2-dependent structural gene expression in response to neural activity. FASEB J. 2009;23:99-106.

39. Tang H, Macpherson P, Marvin M, Meadows E, Klein WH, Yang X-J, Goldman D: A histone deacetylase 4/myogenin positive feedback loop coordinates denervation-dependent gene induction and suppression. Mol Biol Cell. 2009;20:1120-31.

40. Wang Z, Qin G, Zhao TC. HDAC4: mechanism of regulation and biological functions. Epigenomics. 2014;6:139-50.

41. Gerhart J, Neely C, Elder J, Pfautz J, Perlman J, Narciso L, Linask KK, Knudsen K, George-Weinstein M. Cells that express MyoD mRNA in the epiblast are stably committed to the skeletal muscle lineage. J Cell Biol. 2007;178:649-60.

42. Bonnet A, Dai F, Brand-Saberi B, Duprez D. Vestigial-like 2 acts downstream of MyoD activation and is associated with skeletal muscle differentiation in chick myogenesis. Mech Dev. 2010;127:120-36.

43. Hagiwara N. Sox6, jack of all trades: A versatile regulatory protein in vertebrate development. Dev Dyn. 2011;240:1311-21.

44. Tagariello A. Balanced translocation in a patient with craniosynostosis disrupts the SOX6 gene and an evolutionarily conserved non-transcribed region. J Med Genet. 2006;43:534-40.

45. Hagiwara N, Yeh M, Liu A. Sox6 is required for normal fiber type differentiation of fetal skeletal muscle in mice. Dev Dyn. 2007;236:2062-76

46. von Hofsten J, Elworthy S, Gilchrist MJ, Smith JC, Wardle FC, Ingham PW. Prdm1- and Sox6-mediated transcriptional repression specifies muscle fibre type in the zebrafish embryo. EMBO Rep. 2008;9:683-9.

47. Ganfornina MD, Sanchez D, Pagano A, Tonachini L, Descalzi-Cancedda F, Martinez S. Molecular characterization and developmental expression pattern of the chicken apolipoprotein D gene: Implications for the evolution of vertebrate lipocalins. Dev Dyn. 2005;232:191-9

48. Teltathum T, Mekchay S. Proteome changes in Thai indigenous chicken muscle during growth period. Int J Biol Sci. 2009;5:679-85.

49. Li Z. Desmin is essential for the tensile strength and integrity of myofibrils but not for myogenic commitment, differentiation, and fusion of skeletal muscle. J Cell Biol. 1997;139:129-44.

50. Paulin D, Li Z. Desmin: a major intermediate filament protein essential for the structural integrity and function of muscle. Exp Cell Res. 2004;301:1-7.

51. Otten $E$, Asimaki A, Maass $A$, van Langen IM, van der Wal $A$, de Jonge $N$, van den Berg MP, Saffitz JE, Wilde AAM, Jongbloed JDH, van Tintelen JP: Desmin mutations as a cause of right ventricular heart failure affect the intercalated disks. Heart Rhythm. 2010;7:1058-64 
52. Zoltowska K, Webster R, Finlayson S, Maxwell S, Cossins J, Muller J, Lochmuller H, Beeson D. Mutations in GFPT1 that underlie limb-girdle congenital myasthenic syndrome result in reduced cell-surface expression of muscle AChR. Hum Mol Genet. 2013:22:2905-13.

53. Janisch S, Sharifi AR, Wicke M, Krischek C. Changing the incubation temperature during embryonic myogenesis influences the weight performance and meat quality of male and female broilers. Poult Sci. 2015;94:2581-8.

54. Pruitt KD, Brown GR, Hiatt SM, Thibaud-Nissen F, Astashyn A, Ermolaeva O, Farrell CM, Hart J, Landrum MJ, McGarvey KM, Murphy MR, O'Leary NA, Pujar S, Rajput B, Rangwala SH, Riddick LD, Shkeda A, Sun H, Tamez P, Tully RE, Wallin C, Webb D, Weber J, Wu W, DiCuccio M, Kitts P, Maglott DR, Murphy TD, Ostell JM. RefSeq: an update on mammalian reference sequences. Nucleic Acids Res. 2014;42:D756-63.

\section{Submit your next manuscript to BioMed Central and we will help you at every step:}

- We accept pre-submission inquiries

- Our selector tool helps you to find the most relevant journal

- We provide round the clock customer support

- Convenient online submission

- Thorough peer review

- Inclusion in PubMed and all major indexing services

- Maximum visibility for your research

Submit your manuscript at www.biomedcentral.com/submit 